\title{
Prefronto-Striatal Structural Connectivity Mediates Adult Age Differences in Action Selection
}

\author{
Amirhossein Rasooli, ${ }^{1,2 *}$ Hamed Zivari Adab, ${ }^{1,2 *}$ Sima Chalavi, ${ }^{1,2}$ Thiago S. Monteiro, ${ }^{1,2}$ Thijs Dhollander, ${ }^{3}$ \\ ${ }^{-}$Dante Mantini, ${ }^{1,4}$ and ${ }^{\circledR}$ Stephan P. Swinnen ${ }^{1,2}$ \\ ${ }^{1}$ Movement Control and Neuroplasticity Research Group, Department of Movement Sciences, Group Biomedical Sciences, KU Leuven, Leuven 3000, \\ Belgium, ${ }^{2} \mathrm{KU}$ Leuven Brain Institute, Leuven 3000, Belgium, ${ }^{3}$ Murdoch Children's Research Institute, Melbourne, Victoria 3052, Australia, and \\ ${ }^{4}$ Brain Imaging and Neural Dynamics Research Group, Istituto Di Ricovero e Cura a Carattere Scientifico San Camillo Hospital, Venice 30100, Italy
}

In complex everyday environments, action selection is critical for optimal goal-directed behavior. This refers to the process of choosing a proper action from the range of possible alternatives. The neural mechanisms underlying action selection and how these are affected by normal aging remain to be elucidated. In the present cross-sectional study, we studied processes of effector selection during a multilimb reaction time task in a lifespan sample of healthy human adults $(N=89 ; 20-75$ years; 48 males, 41 females). Participants were instructed to react as quickly and accurately as possible to visually cued stimuli representing single-limb or combined upper and/or lower limb motions. Diffusion MRI was used to study structural connectivity between prefrontal and striatal regions as critical nodes for action selection. Behavioral findings revealed that increasing age was associated with slowing of action selection performance. At the neural level, aging had a negative impact on prefrontostriatal connectivity. Importantly, mediation analyses revealed that the negative association between action selection performance and age was mediated by prefronto-striatal connectivity, specifically the connections between left rostral medial frontal gyrus and left nucleus accumbens as well as right frontal pole and left caudate. These results highlight the potential role of prefronto-striatal white matter decline in poorer action selection performance of older adults.

Key words: action selection; anatomical constrained tractography; diffusion imaging; multilimb reaction time; prefrontostriatal connectivity

\section{Significance Statement}

As a result of enhanced life expectancy, researchers have devoted increasing attention to the study of age-related alterations in cognitive and motor functions. Here we study associations between brain structure and behavior to reveal the impact of central neural white matter changes as a function of normal aging on action selection performance. We demonstrate the critical role of a reduction in prefronto-striatal structural connectivity in accounting for action selection performance deficits in healthy older adults. Preserving this cortico-subcortical pathway may be critical for behavioral flexibility and functional independence in older age.

\section{Introduction}

Everyday behavior requires a continuous stream of decisions for actions among the many options emerging from navigation through complex habitats. We need to decide what to do and

\footnotetext{
Received July 2, 2020; revised Nov. 6, 2020; accepted Nov. 10, 2020.

Author contributions: A.R., H.Z.A., D.M., and S.P.S. designed research; A.R. and H.Z.A. performed research; A.R. and H.Z.A. analyzed data; A.R. and H.Z.A. wrote the first draft of the paper; A.R., H.Z.A., S.C., T.S.M., T.D., D.M., and S.P.S. edited the paper; A.R. and H.Z.A. wrote the paper.

${ }^{*}$ A.R. and H.Z.A. contributed equally to this work.

The authors declare no competing financial interests.

This work was supported by Research Foundation Flanders G089818N, Excellence of Science Grant EOS 30446199 (MEMODYN), and the KU Leuven Research Fund (16/15/070. We thank all participants for taking part in this study; and René Clerckx for programming of the behavioral task.

Correspondence should be addressed to Hamed Zivari Adab at Hamed.Zivariadab@kuleuven.be.

https://doi.org/10.1523/JNEUROSCI.1709-20.2020

Copyright $\odot 2021$ the authors
}

how to do it, referring to action selection and action specification, respectively. Often, these processes may operate in an integrated manner (Cisek, 2007). The "what" processes relate to goal establishment (selection of motor goals). The "how" processes refer to composing the movement to achieve that goal (motor planning) (Wong et al., 2015). Motor planning refers to selection of the effector and specification of the required movement. When many potential actions are available, a specific action needs to be chosen among the many motor bids that compete with each other while others are deselected (Humphries, 2014). Action selection plays a major role in the survival of biological systems, motivating its investigation at both behavioral and neural levels (Gurney et al., 1998).

A rich history of experimental work has addressed action (effector) selection processes using reaction time (RT) paradigms (Welford, 1980; Stoloff et al., 2011; Labruna et al., 2014; 
Greenhouse et al., 2015). A simple or complex stimulus is followed by a temporal delay before the buildup of muscle activity and onset of movement. RT can be further decomposed into a central processing (premotor time) and peripheral component (motor time). Reacting to a single stimulus (simple RT [SRT]) is faster than reacting to multiple possible stimuli (choice RT [CRT]), and the difference between CRT and SRT reflects the neural processes underlying action selection (Donders, 1969). Based on this idea, our group introduced a multilimb RT (ML-RT) task, which focuses on visual stimulus processing for effector selection (Boisgontier et al., 2014). It consists of SRT and CRT subtasks requiring the selection and execution of one or more limb movements (1-4 limb segments) (Boisgontier et al., 2014). Because of the strong connectivity between brain regions controlling the limbs, accurate task performance requires a balance between excitatory (effector selection and integration) and inhibitory mechanisms (to suppress the nonselected limb segments) to realize a unified action plan (Swinnen, 2002). Nevertheless, the neural pathways underlying these mechanisms remain elusive and require further investigation.

Cortico-subcortical brain systems contribute to ultimate selection among competing actions. On the one hand, the prefrontal areas process information that is pertinent to action selection (Miller, 2000; Tanji and Hoshi, 2001) and its subjective value (Schultz et al., 2000; Wallis, 2007). On the other hand, the basal ganglia also play a critical role in action selection and inhibition of the competing alternatives, thereby instantiating the desired action plan (Mink, 1996; Redgrave et al., 1999; Schultz, 2004; Colder, 2015). Specifically, the striatum (caudate [CA], putamen, and nucleus accumbens [NAC]) receives inputs from various regions, particularly the (pre)frontal regions, and information about action selection is sent from the basal ganglia via the thalamus to the cortex (Alexander et al., 1986). Although structural connectivity (SC) of the basal ganglia has been studied in detail (Cacciola et al., 2017; Milardi et al., 2019), it is still unclear which specific prefronto-striatal connections are candidate predictors of action selection and how these are affected by aging and disease.

Brain morphologic studies have reported age-related decreases in volume and shape of the basal ganglia and their association with behavioral decline (Serbruyns et al., 2015; Chalavi et al., 2018). Specifically, age-related gray matter atrophy of the CA and NAC has been found to result in longer action selection times during ML-RT task performance (Boisgontier et al., 2016). Moreover, age-associated white matter alterations may compromise the information transmission capacity in prefronto-striatal connections. Interestingly, Bonifazi et al. (2018) used functional (resting-state) and structural (diffusion MRI [dMRI] tractography) connectivity to establish a connectome "age" (structure-function connectome) and compared this with the chronological age. They found that the fronto-striato-thalamic circuit made the strongest contribution to age estimation. Nevertheless, which specific prefronto-striatal connections mediate different behavioral task conditions remains unclear.

Here, we used dMRI to investigate SC of the prefrontalstriatal pathways in association with action selection performance during the ML-RT task in healthy adults (20-75 years). We hypothesized that: (1) older age would result in increases in the central processing component of RT; (2) prefronto-striatal connectivity status would differ among age groups; and (3) these SC differences would mediate the association between RT and age.

\section{Materials and Methods}

Participants. Ninety-two right-handed adults, aged between 20 and 75 years with a normal or corrected-to-normal vision, participated in the study. Three participants were excluded: 2 participants scored $<24$ on the Montreal Cognitive Assessment test (Nasreddine et al., 2005) and 1 participant exhibited a brain lesion, as identified on their MRI scan and verified by a trained clinical radiologist. Thus, the final analyses were performed on the remaining 89 participants (age, mean \pm SD: $48.16 \pm 17.44$ years, 48 males) consisting of 29 young adults (YA): from $20-40$ years, $26.96 \pm 6.02$ years, 13 males; 31 middleaged adults (MA): from 40-60 years, $49.50 \pm 5.70$ years, 17 males, and 29 older adults $(\mathrm{OA}):>60$ years, $67.94 \pm 3.46$ years, 18 males. None suffered from psychiatric and/or neuromuscular impairments. These subgroups were only applied for the behavioral analysis using ANOVA. In the remaining analyses, age was considered a continuous variable. The study was approved by the local Medical Ethics Committee of KU Leuven (study number S60428) in accordance with the Declaration of Helsinki and its amendments. All participants provided written informed consent before the start of the study and received financial compensation for participating.

$M L-R T$ task. The ML-RT task has been fully described in previous publications (Boisgontier et al., 2014, 2016). Generally, this task has proven to be very instrumental in uncovering interactions between brain structure and behavior in general and processes of motor facilitation and inhibition during complex multilimb task performance specifically (Boisgontier et al., 2014). Participants were seated in front of a PC screen, with their forearms resting on a table and their hands and forefeet on tablets with capacitive proximity switches (Fig. 1A; Pepperl Fuchs CBN5F46-E2, sampling frequency: $1000 \mathrm{~Hz}$ ). Four squares representing the four limb segments were presented on the screen. Mapping of the visual stimuli was maximally congruent with the location of the effectors; that is, the right and left upper squares represented the right and left hands, whereas the right and left lower squares represented the right and left feet, respectively. Once all four limb segments were in contact with the tablets simultaneously, a subset of the squares on the screen turned blue depending on the limbs to be moved. In response to this stimulus, participants had to release contact with the corresponding tablet(s) as quickly and as correctly as possible by lifting the indicated limb segment(s). The detailed sequence for each trial was as follows (Fig. 1B): Squares were gray when limbs were not in contact with the tablets. They turned white when the limb segments contacted the corresponding tablet. A trial started as soon as the four limbs were in contact with the tablets simultaneously. After a randomly varying time interval, ranging from 500 to $1000 \mathrm{~ms}$, one or more squares turned blue and the participant had to release contact with the corresponding tablet(s) as quickly as possible. If the participant lifted the incorrect $\operatorname{limb}(\mathrm{s})$, the corresponding square(s) turned red. If the participant lifted the correct $\operatorname{limb}(\mathrm{s})$, the corresponding square(s) turned green. A trial was only validated when the response was fully correct (i.e., without any red squares on the screen). Once the trial was validated, the green squares turned back to gray. Then, the participant repositioned all limb segments on the tablets to start a new trial. All the 15 possible stimulus modes were tested: 4 single-limb conditions, 6 two-limb conditions, 4 three-limb conditions, and 1 four-limb condition. These conditions were grouped into 6 stimulus clusters based on the number of recruited limbs and the coupling/decoupling interactions involved (Fig. 1C): 1-limb stimulus cluster (1L); 2-homologous upper or lower limb stimulus cluster (2L-Homo); 2-ipsilateral (right upper right lower or left upper left lower) limb stimulus cluster (2L-Ipsi); 2-diagonal (left upper right lower or left lower right upper) limb stimulus cluster (2L-Diag); 3-limb (2L-Ipsi plus upper/lower contralateral) stimulus cluster (3L); and one 4-limb cluster (4L). The differential difficulty of these coordination modes is not necessarily determined by the number of limb segments that have to be moved but also by their degree of convergence with or reliance on preexisting preferred coordination modes (Swinnen, 2002). For example, homologous limb combinations are easy to perform because these are consistent with the default coordination modes (i.e., moving either both upper or both lower limbs together). Conversely, performing 2-limb diagonal coordination modes are more difficult because they require suppression of the homologous counterpart. For example, 


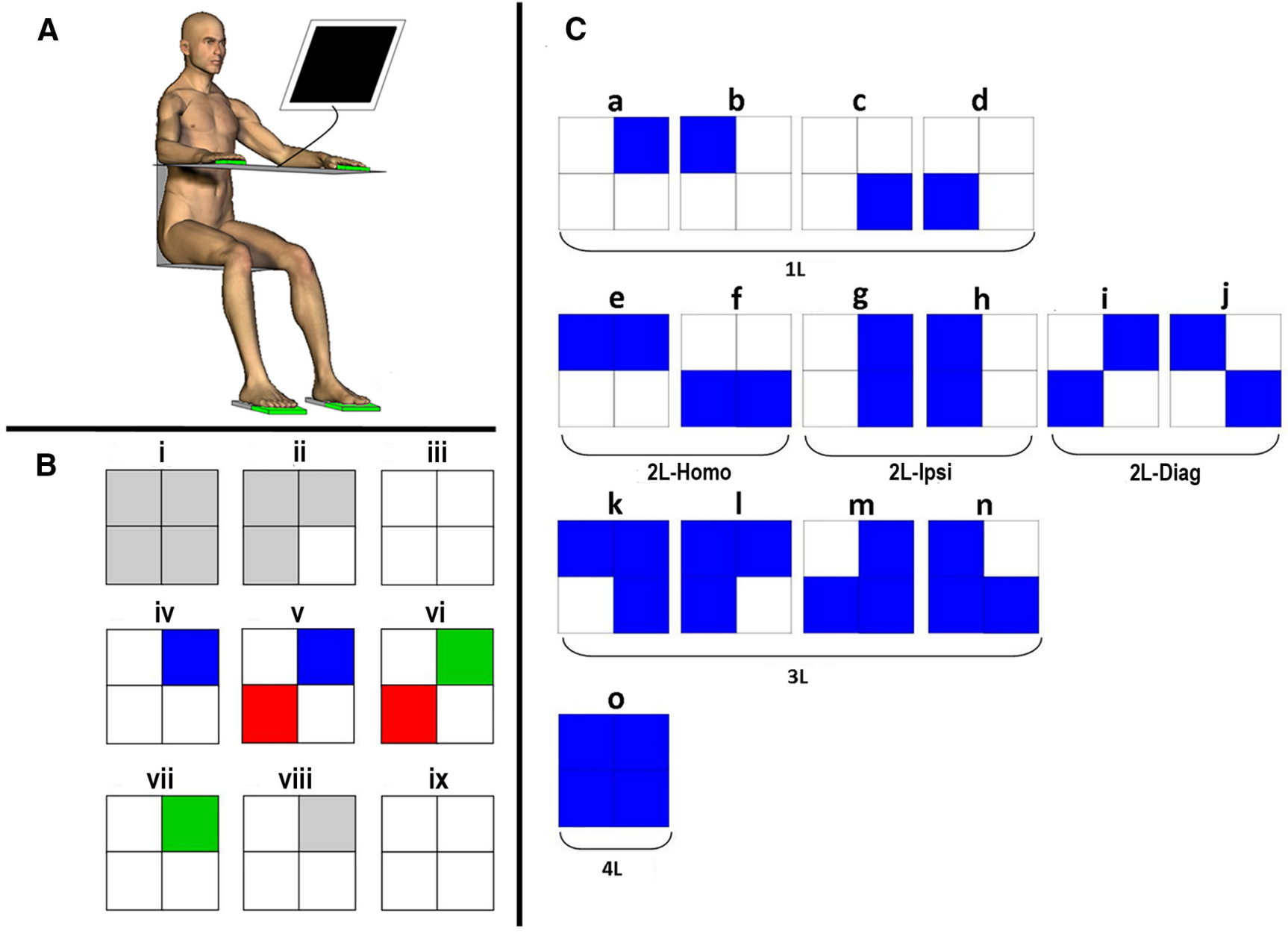

Figure 1. A, Experimental setup. Participants are seated in front of a screen, with their forearms resting on a table and their fingers and forefeet on tablets. $\boldsymbol{B}$, Exemplar ML-RT task trial procedure. Top squares represent stimulus cues for left and right hand. Bottom squares represent cues for left and right feet, respectively. $\boldsymbol{B} \boldsymbol{i}$, When the limb segments are not connected to the tablets, the corresponding squares are gray. Bii, They turn white as soon as the corresponding limbs contact the tablets. Biii, The setup is ready for a trial when all of the limbs are in contact with their corresponding tablets. Biv, After a randomly varying time ranging from 500 to $1000 \mathrm{~ms}$, the stimuli are presented by blue squares, indicating the limbs that should be released as quickly and correctly as possible. $\boldsymbol{B v}$, If the participant releases the incorrect limb, the corresponding square(s) turn(s) red. Bvi, If the participant lifts the correct limb(s), the corresponding square(s) turn(s) green. Bvii, A trial is not validated until the response is fully correct, that is, without any red square on the screen. Bviii, As soon as the trial is validated, the green squares turn back to gray. Bix, Participants have to reposition all limb segments on the tablets to start a new trial. C, Different stimulus modes and clusters: the 15 possible modes ("a" to " 0 ") are grouped into 6 clusters (1L, 2L-Homo, 2L-Ipsi, 2L-Diag, 3L, and 4L) based on the number of limbs to be recruited (1, 2, 3, or 4) and the coupling/decoupling interactions involved. Adapted with permission from Boisgontier et al. (2014).

moving the right arm together with the left leg requires suppression of the left arm and right leg movement given the pervasive coupling tendency between the homologous limbs (for review, see Swinnen, 2002). Thus, depending on the stimulus condition, active limb selection is also accompanied by deselection/suppression of other limbs. Together, this combination of excitation and inhibition mechanisms impacts the time required to perform these ML-RT tasks.

For familiarization purposes, participants were instructed to perform each stimulus mode before initiation of the experiment. The experiment was composed of two blocks, which were 5 min apart. The first block, called SRT, was composed of randomized 10 trial runs of each of the 15 stimulus modes. Before each run, the participant was presented with a figure of the stimulus that would appear on the screen for the next 10 trials (predictive: no choice required). The second block, called CRT, consisted of performing 150 trials (15 stimulus modes $\times 10$ trials) in randomized order (nonpredictive: choice required). In total, each participant performed 300 trials.

Behavioral data analysis. For error-free trials, the elapsed time between the onset of visual stimulus presentation and the time the participant released the corresponding limbs was obtained and defined as $\mathrm{RT}$. We chose to include only error-free trials in the RT analyses because including erroneous trials would require the addition of a correction time to the elapsed RT, which would detract from and complicate the interpretation of the true action selection time. Within each stimulus mode of each participant, trials with RT values $>3$ scaled median absolute deviations (Leys et al., 2013) were considered as outliers and removed. This procedure resulted in removing $5.5 \pm 2.9 \%$ (mean \pm SD) of the trials across all participants. The median value of the remaining trials in each stimulus mode was obtained and averaged to summarize the initial 15 stimulus modes into 6 stimulus clusters (1L, 2L-Homo, 2LIpsi, $2 \mathrm{~L}-\mathrm{Diag}, 3 \mathrm{~L}$, and $4 \mathrm{~L}$ ). This procedure was applied to both SRT and CRT trials. Since this study was focused on cognitive processes involved in action selection, the net RT (NRT) was calculated by subtracting the SRT values from the CRT values to provide a distinct measure of the central processing of action selection, free of confounds.

Additionally, the percent error rate in each coordination mode was calculated by dividing the number of error trials by the total number of trials, multiplied by 100. A trial was considered as erroneous when the participant released an incorrect limb. Similar to RT, the simple error percentage values were subtracted from the choice percentage values to obtain the net error rate (NER). Given the presence of more potential error locations in stimulus clusters with the lower number of recruited limbs than those with the higher number of recruited limbs (i.e., three wrong effectors are possible to be recruited in $1 \mathrm{~L}$ stimulus cluster, 2 in 
$2 \mathrm{~L}$, and 1 in $3 \mathrm{~L}$ ), the NERs were divided by 3,2 , and 1 in $1 \mathrm{~L}, 2 \mathrm{~L}$, and $3 \mathrm{~L}$ stimulus clusters, respectively. This adjustment makes the NER comparison across the stimulus clusters more meaningful (Boisgontier et al., 2014, 2016).

Image acquisition. MRI data were acquired using a Philips Achieva 3T scanner with a 32-channel head coil, located at the University Hospital of Leuven, Belgium. The multishell dMRI data were acquired using a spin-echo EPI sequence with the following parameters: $b=700$ $\mathrm{s} / \mathrm{mm}^{2}$ (16 directions); $\mathrm{b}=1200 \mathrm{~s} / \mathrm{mm}^{2}$ (30 directions); $\mathrm{b}=2800 \mathrm{~s} / \mathrm{mm}^{2}$ (50 directions); 6 interleaved volumes without diffusion weighting $(b=0$ $\left.\mathrm{s} / \mathrm{mm}^{2}\right)$; voxel size $=2.5 \times 2.5 \times 2.5 \mathrm{~mm}^{3}, \mathrm{TE} / \mathrm{TR}=74 / 9000 \mathrm{~ms}$; $\mathrm{SENSE}=$ 2; matrix size $=96 \times 96$; and number of axial slices $=50$. In addition, 1 $\mathrm{b}=0 \mathrm{~s} / \mathrm{mm}^{2}$ image was acquired with reversed phase encoding, for the purpose of susceptibility-induced distortion correction. The total acquisition time was $17.5 \mathrm{~min}$. Furthermore, a high-resolution three-dimensional T1-weighted anatomic image was acquired (three-dimensional transient field echo; $\mathrm{TR}=9.6 \mathrm{~ms}$; $\mathrm{TE}=4.6 \mathrm{~ms}$; inversion time $=900 \mathrm{~ms}$; flip angle $=8^{\circ}$; voxel size $=0.98 \times 0.98 \times 1.2 \mathrm{~mm}^{3}$; FOV $=250 \times 250 \times$ $192 \mathrm{~mm}^{3} ; 160$ coronal slices, total scan time $=6 \mathrm{~min}$ ).

Image processing. Where not stated explicitly, processing was performed using the MRtrix3 software package (Tournier et al., 2019). In brief, dMRI data were denoised (Veraart et al., 2016) and corrected for eddy current distortions, motion, and susceptibility-induced distortions (Andersson et al., 2003, 2016; Andersson and Sotiropoulos, 2016). Afterward, 3-tissue response functions representing single-fiber white matter, gray matter, and CSF were obtained from the data themselves using an unsupervised approach (Dhollander et al., 2016). Next, 3-tissue constrained spherical deconvolution was performed for each participant, using averaged (across all participants) response functions for each tissue type with the multishell multitissue constrained spherical deconvolution algorithm (Jeurissen et al., 2014), resulting in the white matter fiber orientation distribution (FOD) for each voxel. Joint bias field correction and global intensity normalization of the 3 -tissue parameters were performed in the log-domain.

Following the initial processing, tractograms were generated. Thus, for each participant, the second-order integration over FOD algorithm (iFOD2) (Tournier et al., 2010) and the anatomically constrained tractography (Smith et al., 2012), with dynamic seeding (Smith et al., 2015b), FOD amplitude threshold 0.06 , step size of $1.25 \mathrm{~mm}$, length of 5-250 $\mathrm{mm}$, and backtracking (Smith et al., 2012) were used to generate 10 million probabilistic streamlines. Furthermore, each streamline was assigned a weight, computed using the spherical-deconvolution informed filtering of tractograms (SIFT2) (see Smith et al., 2015b). Based on each participant's tractogram, an individual connectome was computed using 84 regions (Desikan et al., 2006) parcellated in native space (cortex and cerebellum were parcellated using Freesurfer (Fischl, 2012); subcortical regions were determined using FSL FIRST (Patenaude et al., 2011; see Smith et al., 2015b)), with connection strengths calculated by summing the weights of the relevant streamlines. Intraregional connection strengths were set to zero (Rubinov and Sporns, 2010). The 96 connections/tracts of interest between the striatal node and the PFC were selected for further analysis (Fig. 2). The striatal nodes include the following: the left/right CA (L/R CA), left/right putamen (L/R PU), and left/ right NAC (L/R NAC). The nodes of the PFC include the following: the left/right caudal anterior cingulate gyrus (L/R CACG), left/right caudal middle frontal gyrus (L/R CMFG), left/right lateral orbitofrontal gyrus (L/R LOFG), left/right medial orbitofrontal gyrus (L/R MOFG), left/right rostral anterior cingulate gyrus (L/R RACG), left/right rostral middle frontal gyrus (L/R RMFG), left/right superior frontal gyrus (L/R SFG), and left/right frontal pole (L/R FP).

Total intracranial volume (TIV) was obtained from the T1-weighted image using the standard pipeline of FreeSurfer (Fischl, 2012), and the average participant's head movement across dMRI volumes was computed from the restricted root-mean-square movement output by the FSL (Jenkinson et al., 2012).

Statistical analysis. To investigate age-related differences in the cognitive cost associated with a particular stimulus cluster, the NRT and NER were separately modeled in Statistica using a $3 \times 5$ repeated-measures ANOVA. In this analysis, age (20-75 years) was considered as a categorical, between-subject factor with three levels: YA, 20-40 years, $n=29$; MA, $40-60$ years, $n=31$, and OA: $>60$ years, $n=29$. Stimulus cluster was considered as a within-subject factor with five levels: $1 \mathrm{~L}, 2 \mathrm{~L}$ Homo, 2L-Ipsi, 2L-Diag, and 3L. The 4L stimulus cluster was not considered in the analyses because: (1) the data from several participants were missing; and (2) this condition always resulted in an absence of error as all limbs had to be recruited. For all the statistical analyses, the significance level was considered as $p<0.05$, two-tailed. $p$ values of ANOVAs were corrected for sphericity using the Greenhouse-Geisser method when Mauchly's test was significant. Following the statistical method reported previously (Boisgontier et al., 2016), error rates were transformed using the $(\mathrm{NER}+2.5)^{1 / 2}$ transformation (Bartlett, 1947) to stabilize the variance of the measurements and increase the validity of the analysis. When ANOVA revealed significant effects, post hoc tests (Tukey HSD with correction for multiple comparisons) were used to identify the loci of the effects. Partial $\eta$ squared $\left(\eta^{2} \mathrm{P}\right)$ were reported to indicate small (0.01), medium (0.06), and large (0.14) effect sizes (Sink and Stroh, 2006).

To investigate associations between age (as a continuous variable) or action selection performance (NRT or NER) and prefronto-striatal SC, nonparametric Spearman correlation analyses were conducted in MATLAB R2019b (The MathWorks). In these analyses, gender, TIV, and the average participant head movement across dMRI volumes were controlled for. $p$ values were corrected for multiple comparisons using the false discovery rate (FDR) method with $q=0.05$ (Benjamini and Hochberg, 1995).

Mediation analysis. To investigate whether declines in prefrontostriatal SC with aging contribute to the age-related slowing in multilimb action selection time, mediation analyses were performed (MacKinnon et al., 2007). In these analyses, mediation of the age-action selection time relation by white matter SC was conceptually defined as age negatively affecting SC, and declined white matter SC leading to worse performance (i.e., increased action selection time). Thus, connections/tracts exhibiting statistically significant (after FDR correction) negative associations with both age and NRT were selected as candidate mediators.

The commonly used mediation model (Baron and Kenny, 1986), implemented in the M3 mediation toolbox (Wager et al., 2008, 2009), was used. The first step in this approach is the evaluation of the overall effect of age on action selection performance, hereafter referred to as total effect of age on action selection (c) (Fig. 3). Next, we evaluated whether (part of) this total effect of age on action selection performance was explained by the effect of age on SC of a specific white matter tract (i.e., mediator). Accordingly, for each tract of interest, a mediation model was tested in which the total effect was partitioned into two distinct pathways that were evaluated using ordinary least squares path analysis. The first pathway, hereafter referred to as indirect pathway $(a b)$, represents how age contributes to differences in the candidate mediator variable, and how this in turn contributes to differences in action selection performance. The indirect effect was estimated as $a b$, meaning the product of path $a$, which represents the effect of age on the mediator variable, and path $b$, which represents the effect of the mediator variable on action selection performance while controlling for the effect of age. Of particular interest was the indirect effect, since a significant indirect effect would indicate significant mediation by the mediator variable used in the model. Although not critical for the evaluation of the significance of mediation by the mediator variable being tested, the second pathway, hereafter referred to as direct pathway $\left(c^{\prime}\right)$, was also investigated. This pathway represents the residual influence of age on action selection performance, that is, the effect of age on action selection performance independent of its effect via the mediator variable (i.e., tract SC) used. This direct effect was estimated with $c^{\prime}$, and corresponds exactly to the difference between the total and the indirect effect of age on action selection performance in the model. One of the main advantages of this method is that it enables direct testing of the significance of the indirect effect and therefore mediation. Here, this was accomplished using 10,000 bootstrap samples to determine bias-corrected 95\% CIs for the indirect effects. Accordingly, indirect effects with 95\% CIs entirely above zero (one-tailed test based on the directional 

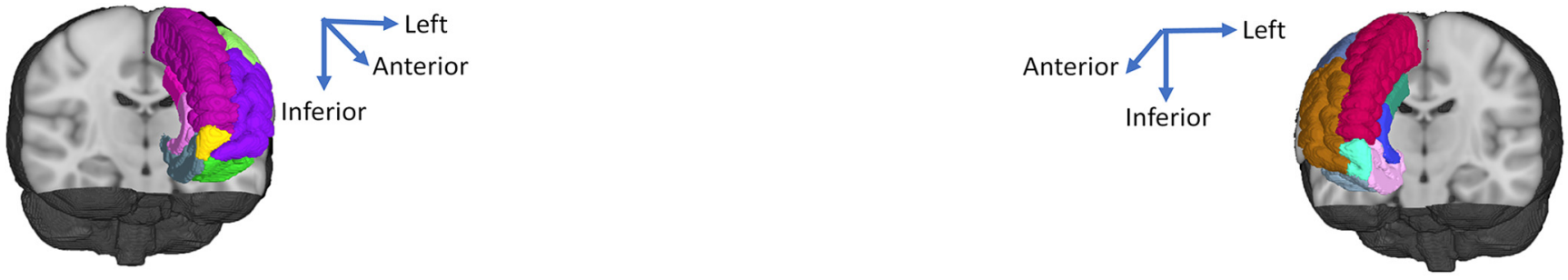

left (pre)frontal regions

right (pre)frontal regions
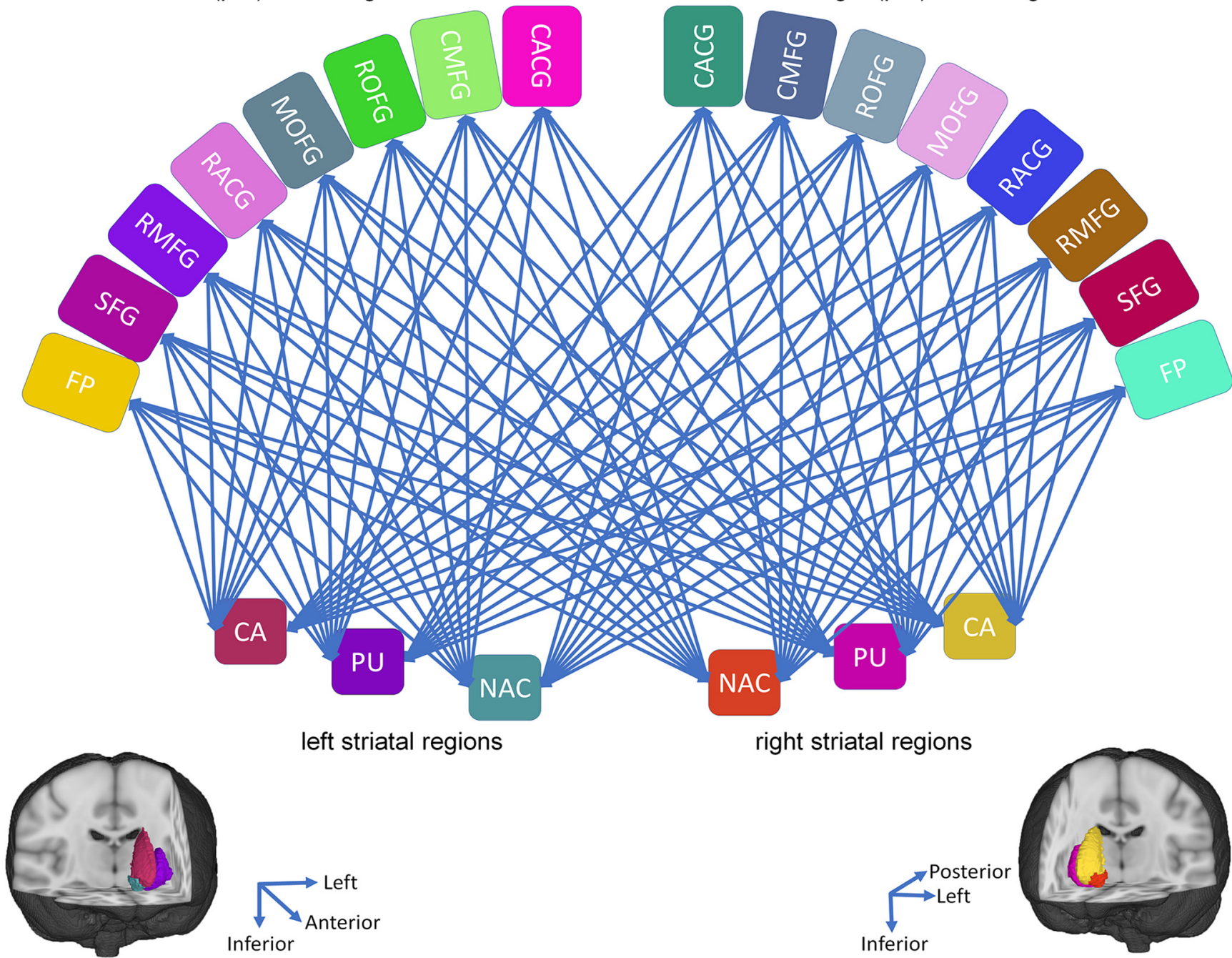

right striatal regions

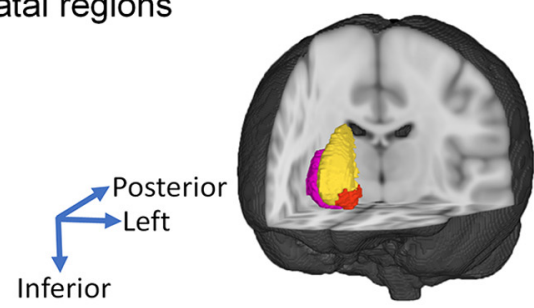

Figure 2. Representation of prefronto-striatal regions and corresponding connections evaluated in this study. CACG = caudal anterior cingulate gyrus, CMFG $=$ caudal middle frontal gyrus, LOFG = lateral orbitofrontal gyrus, MOFG = medial orbitofrontal gyrus, RACG = rostral anterior cingulate gyrus, RMFG = rostral middle frontal gyrus, SFG = superior frontal gyrus, FP = frontal pole, $\mathrm{CA}=$ caudate, $\mathrm{PU}=$ putamen, $\mathrm{NAC}=$ nucleus accumbens.

hypotheses) were regarded as significantly mediating the relation between age and action selection performance.

\section{Results}

\section{ML-RT task}

With regard to the temporal duration of the action selection process (NRT), results of the two-way $3 \times 5$ ANOVA demonstrated a main effect of stimulus cluster $\left(F_{(1.55,133.66)}=263.62 ; p<0.001\right.$; $\left.\eta_{\mathrm{P}}^{2}=0.75\right)$. Post hoc analyses showed no difference in NRT between the 2L-Diag and 3L stimulus clusters $(p=0.082$; see Fig. $4 A$ ). The NRT was the longest for these stimulus clusters and decreased progressively in the $2 \mathrm{~L}-\mathrm{Ipsi}>2 \mathrm{~L}-\mathrm{Homo}>1 \mathrm{~L}$ stimulus clusters, respectively ( $p$ values $<0.001$ ). These findings indicate a slowing of multilimb action selection times as the task becomes more complex. There was also a significant main effect of age $\left(F_{(2,86)}=23.76 ; p<0.001 ; \eta_{\mathrm{P}}^{2}=0.36\right)$. Post hoc analyses indicated that NRT was the longest in the OA group, followed by the MA group and finally the YA group that showed the shortest time ( $p$ values $<0.006)$. This suggests that multilimb action selection is already compromised as early as at middle age. Finally, the significant age $\times$ stimulus cluster interaction effect $\left(F_{(3.11,133.66)}=\right.$ 6.47; $\left.p<0.001 ; \eta_{\mathrm{P}}^{2}=0.13\right)$ indicates that age disproportionally affected the action selection times in the more difficult compared with easier stimulus clusters.

With regard to error rate (NER), the two-way ANOVA demonstrated a main effect of stimulus cluster $\left(F_{(2.88,247.73)}=163.57\right.$; $\left.p<0.001 ; \eta_{\mathrm{P}}^{2}=0.66\right)$. Post hoc analyses revealed that error rates 


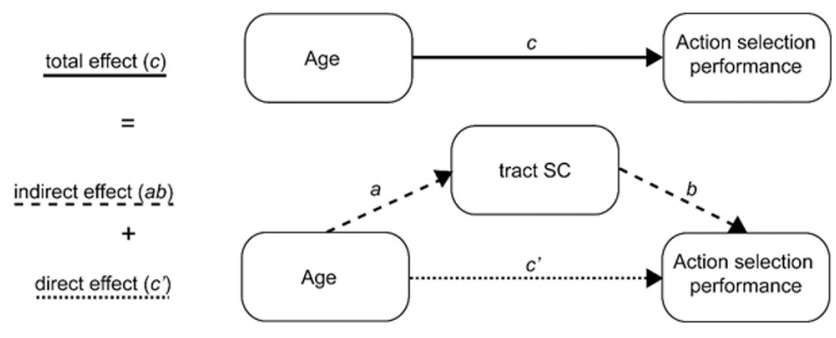

Figure 3. Mediation analysis. The mediation model used to determine whether the agerelated differences in multilimb action selection performance are mediated by alterations in tract SC. c, Total effect of age on action selection performance (solid line). This total effect was, per tract of interest, separated into two distinct pathways: an indirect (mediation) effect ( $a b$, dashed arrows): with a reflecting the effect of age on the mediator variable "tract SC" and $b$ reflecting the effect of the mediator variable "tract $S C^{\prime}$ on action selection performance while controlling for age; and a direct effect $\left(c^{\prime}\right.$, dotted arrow) of age on action selection performance, that is, the effect of age on action selection performance independent of its effect through the mediator variable "tract SC."

were the highest in the $2 \mathrm{~L}-\mathrm{Diag}$ and $3 \mathrm{~L}$ stimulus clusters $(p$ values $<0.001$; see Fig. $4 B$ ). However, NER did not differ between these two stimulus clusters $(p=0.12)$. NER did not differ between $1 \mathrm{~L}$ and $2 \mathrm{~L}-\mathrm{Homo}$ stimulus clusters $(p=0.2)$, and the $2 \mathrm{~L}$ Ipsi stimulus cluster had the lowest NER among all stimulus clusters $(p$ values $<0.026)$. The main effect of age $\left(F_{(2,86)}=1.55\right.$; $\left.p=0.22 ; \eta_{\mathrm{P}}^{2}=0.04\right)$ and the interaction effect of age $\times$ stimulus cluster $\left(F_{(5.76,247.73)}=1.3 ; p=0.26 ; \eta_{\mathrm{P}}^{2}=0.03\right)$ were not significant.

\section{Brain-behavior and age association}

NRTs were longer in 2L-Diag and 3L stimulus clusters and shorter in the 1L, 2L-Homo, and 2L-Ipsi clusters. Accordingly, analogous to previous studies (Boisgontier et al., 2014, 2016), brain-behavior analyses were performed on 2 levels of task complexity: difficult (average of 2L-Diag and 3L) and easy (average of 1L, 2L-Homo, and 2L-Ipsi) task conditions. The results of the correlation analyses between prefronto-striatal connectivity and NRT in these collapsed task conditions are shown in Figure 5.

Regarding the easy task condition (Fig. 5A), negative associations between NRT and SC were found for the left NAC-left RMFG, left NAC-left FP, right NAC-right RMFG, and right NAC-right FP tracts (all Spearman's $\rho<-0.33$; all FDR-corrected $p$ values $<0.002$ ). Regarding the difficult task condition (Fig. $5 B$ ), the connectivity of the right FP-right/left CA was negatively associated with NRT (all Spearman's $\rho<-0.36$; all FDR-corrected $p$ values $<0.001)$. The negative direction of these correlations indicates that weaker SC was associated with increased (slower) action selection times. It is also worth noting that, while the spatial extent of these associations is confined to intrahemispheric connections for the easy condition, it also includes the interhemispheric connections for the difficult condition. The same analysis was performed on NER, but no significant associations were obtained.

Age-related differences in prefronto-striatal white matter connectivity were also observed (Fig. 6). The negative associations of connectivity with age, reflecting decreased connectivity with advancing age, were mainly pronounced for the CA connections to the prefrontal regions (both intrahemispheric and interhemispheric). Except for left CACG, the connectivity of all left prefrontal areas to right CA showed significant negative associations with age (all Spearman's $\rho<-0.41$; all FDR-corrected $p$ values $<0.01)$. Similarly, right CA-right RMFG/SFG/FP connections demonstrated significant negative associations with age (all Spearman's $\rho<-0.48$; all FDR-corrected $p$ values $<0.01)$. For the left CA, left CA-left LOFG/RMFG/SFG/FP/right MOFG/FP showed significant negative associations with age (all Spearman's $\rho<-0.43$; all FDR-corrected $p$ values $<0.01)$. Age-related decreases in connectivity were not limited to the CA. For example, connectivity of the right NAC-right FP and left NAC-left RMFG/ FP/right RACG decreased with age (all Spearman's $\rho<-0.35$; all FDR-corrected $p$ values $<0.01)$. Conversely, a significant increase in connectivity with age was seen for the white matter connections between the PU and ipsilateral MOFG in both hemispheres (both $r$ values $>0.33$; both FDR-corrected $p$ values $<0.01$ ).

In general, increasing age was mainly associated with decreased prefronto-striatal SC. This effect was more prominent for the connections of prefrontal regions to right CA. Only the MOFG connection to PU demonstrated a positive association with age. Together, this demonstrates a widespread and quite homogeneous aging effect on the white matter prefronto-striatal connectivity.

\section{Mediation analysis}

Comparing the results obtained from the brain-behavior correlational analyses in relation to age, it became clear that, except for the right RMFG-right NAC, all prefronto-striatal connections demonstrating associations with NRT were also affected by age. Thus, to further investigate the age-brain-behavior interrelation, mediation analyses were conducted. Accordingly, 3 connections (left NAC-left RMFG, left NAC-left FP, and right NAC-right FP) in the easy and 2 connections (left CA-right FP and right CAright FP) in the difficult task condition were used as candidate mediators of the effect of age on NRT.

The results of these analyses (Fig. 7) revealed significant mediation of the relation between age and NRT in the easy condition through the SC of left NAC-left RMFG (mean \pm SE; $a b=0.41 \pm 0.24, p=0.03, \mathrm{CI}=[0.03,0.96])$. Additionally, the left CA-right FP mediated significantly the relation between age and NRT in the difficult condition $(a b=1.15 \pm 0.69, p=0.0343, \mathrm{CI}=$ $[0.09,2.97])$. Notably, the significant direct path $c^{\prime}$ in all tested models indicated that the achieved mediation was "partial" rather than "full." Conversely, neither the SC of left NAC-right $\mathrm{FP}(a b=0.34 \pm 0.20, p=0.06, \mathrm{CI}=[-0.01,0.74])$ nor the connectivity of right NAC-right FP $(a b=0.16 \pm 0.18, p=0.20, \mathrm{CI}=$ $[-0.11,0.60])$ mediated significantly the effect of age on NRT in the easy condition. Furthermore, the connectivity of right CAright FP did not significantly mediate the relation between age and NRT in the difficult condition $(a b=0.29 \pm 1.18, p=0.80$, CI $=[-2.11,2.53])$.

Together, the results indicate that the increased (slower) NRT in the easy condition because of aging was partially mediated by the reduced left NAC-left RMFG connectivity. Similarly, the agerelated increase in NRT in the difficult condition was partially mediated by the reduced left CA-right FP connectivity.

\section{Discussion}

We investigated the association between prefronto-striatal SC and action selection performance across the lifespan. From a behavioral perspective, aging was accompanied by a significant slowing of action selection time (NRT), whereas NER did not significantly differ. Moreover, increasing task complexity resulted in a significant increase in NRT as well as NER. At the neural level, dMRI revealed a significant aging effect on prefronto-striatal SC. Additionally, SC between the CA and the FP as well as between 

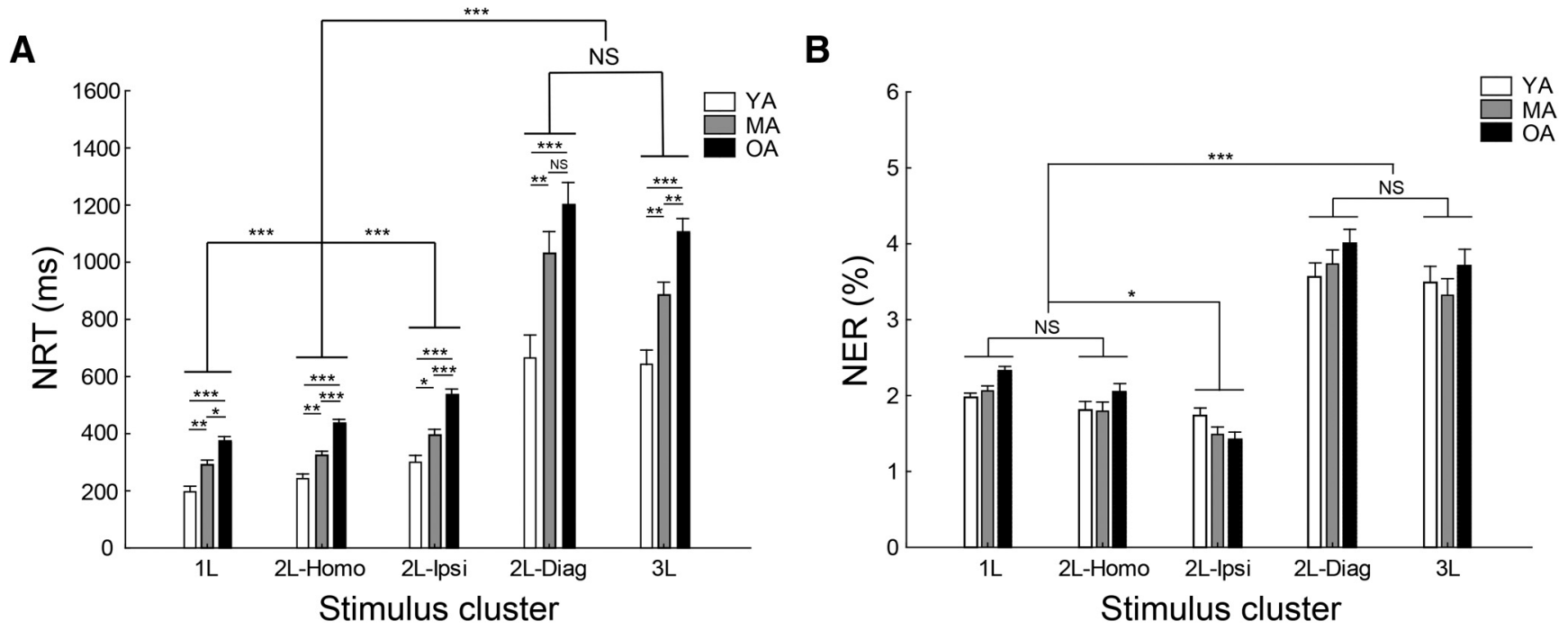

Figure 4. ML-RT task performance: $(\boldsymbol{A})$ NRT and $(\boldsymbol{B})$ transformed NER as a function of stimulus cluster and age group. Bar (error bar) indicates mean (SE). $* p$ corrected $<0.05 . * * p$ corrected $<0.01$. $* * * p$ corrected $<0.001$. NS: Not Significant.

A

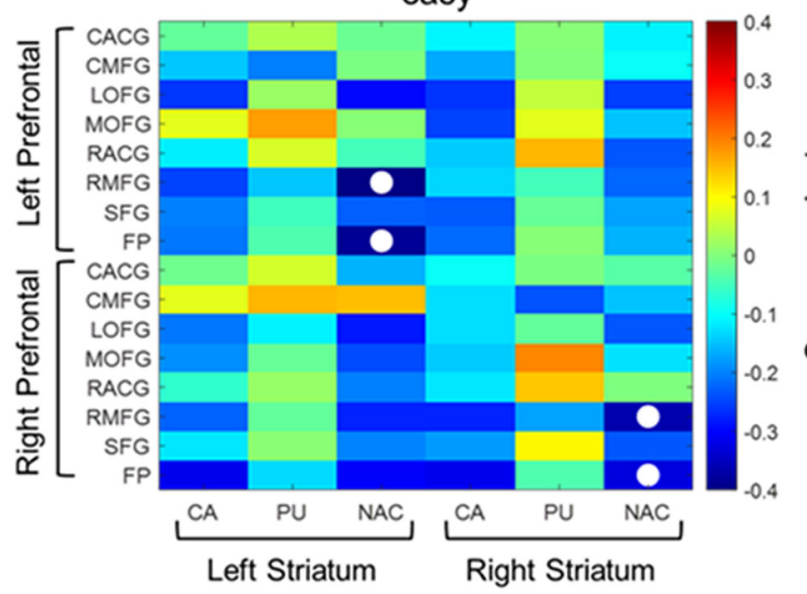

B

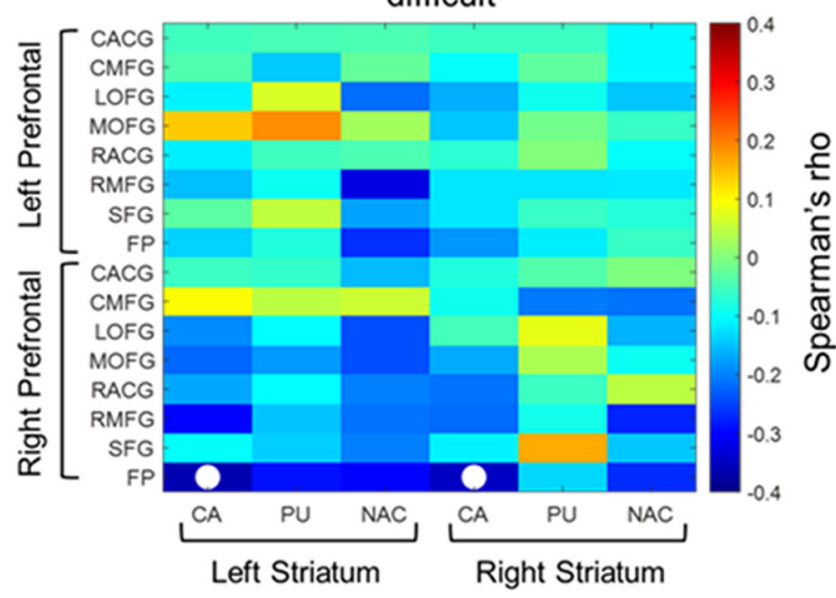

C

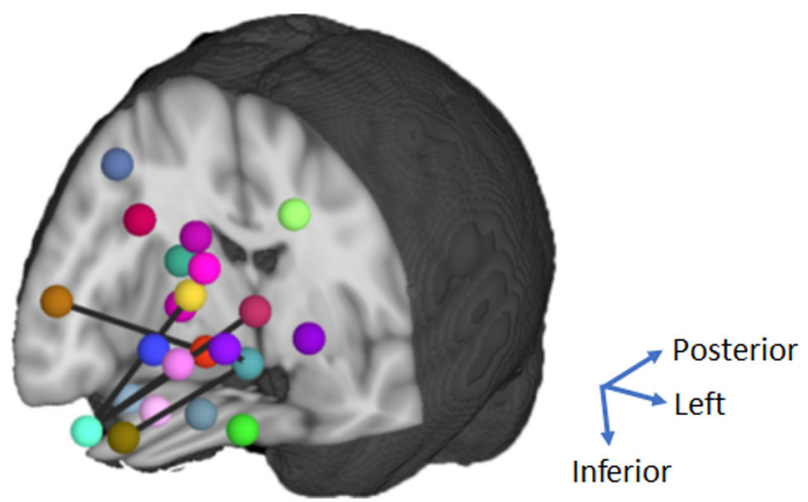

Figure 5. The association of prefronto-striatal SC with NRT in (A) easy (1L, 2L-Homo, and 2L-Ipsi) and (B) difficult (2L-Diag and 3L) task conditions. Colors represent the Spearman's $\rho$ values controlled for gender, TIV, and head movements during dMRI scans. White circles represent significant correlations after FDR correction. $C$, Representation of edges with significant association with behavior overlaid on the $\mathrm{T} 1 \mathrm{MNI}$ space, radiologic orientations. Colors are the same as in Figure 2 .

NAC and rostral medial frontal gyrus showed significant associations with RT in both easy and difficult task conditions. Moreover, left NAC-left RMFG and left CA-right FP SC mediated the effect of age on behavioral performance decline.
Effects of age on action selection behavior

We observed a significant increase in action selection time across the three age groups. The increase in NRT was already evident in the middle-aged group, extending previous findings in which 
A

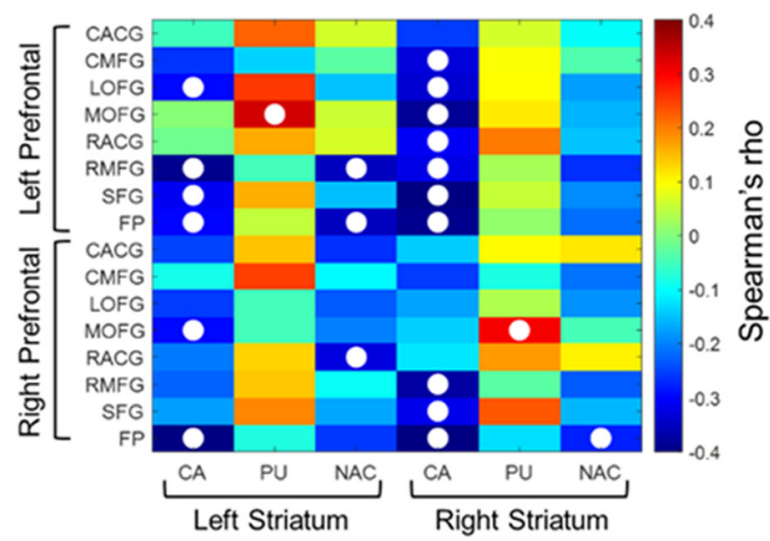

B

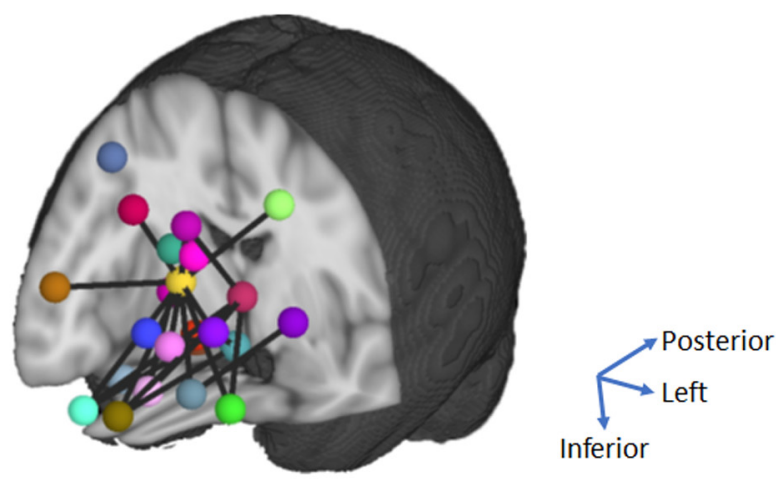

Figure 6. $\boldsymbol{A}$, The association of prefronto-striatal SC with age. Colors represent the Spearman's $\rho$ values controlled for gender, TIV, and head movements during dMRI scans. White circles represent significant correlations after FDR correction. $\boldsymbol{B}$, Connections with significant association with age overlaid the T1 MNI space, radiologic orientations. Colors are the same as in Figure 2.
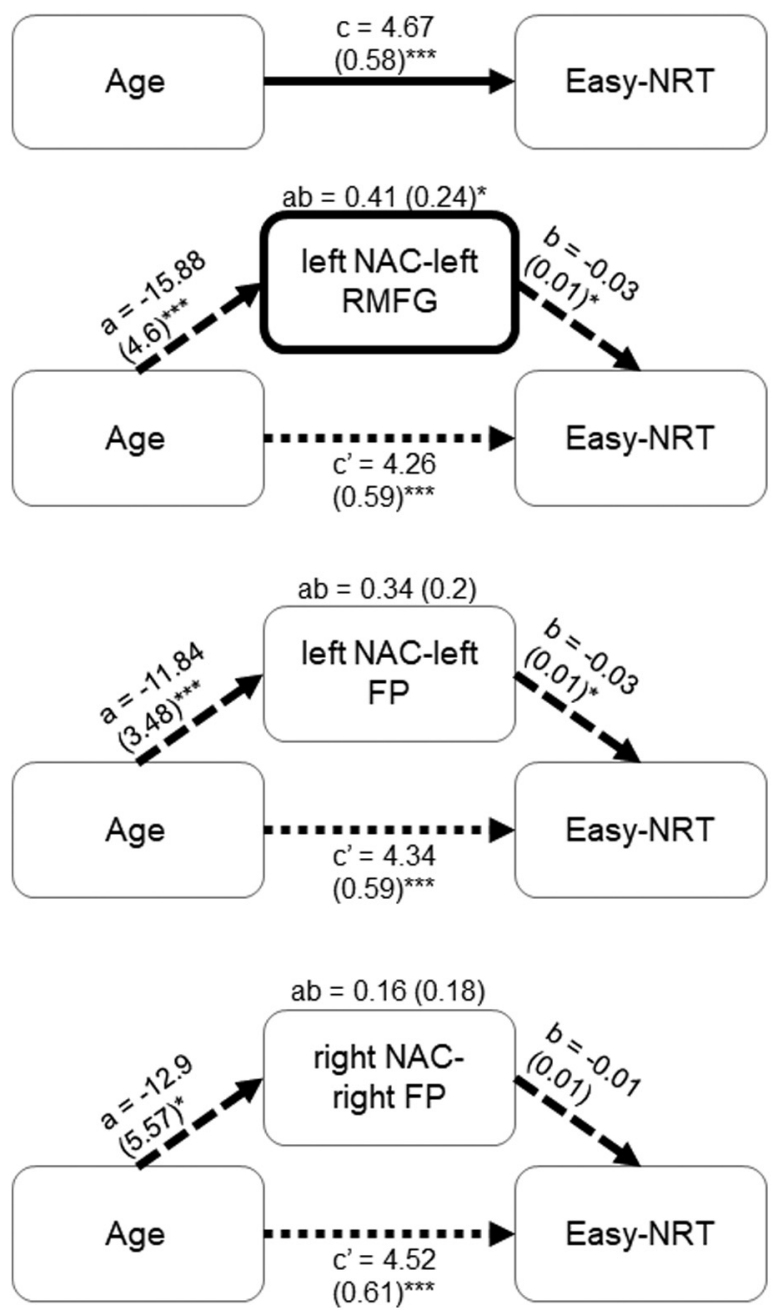
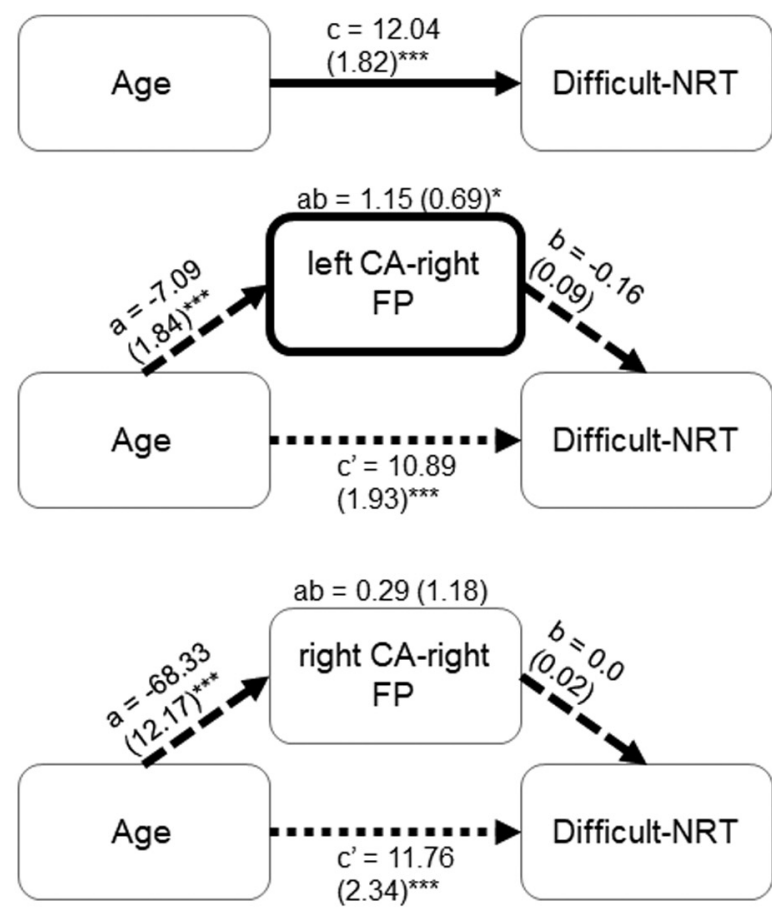

Figure 7. Standard three-path mediation analysis results. Connectivity of left NAC-left RMFG $(a b=0.41 \pm 0.24, p=0.03, \mathrm{Cl}=[0.03,0.96])$ and left CA-right FP $(a b=1.15 \pm 0.69, p=0.03, \mathrm{Cl}=[0.09,2.97])$ significantly mediates the relation between age and NRT in the easy and difficult condition, respectively. $c$, Total effect (solid line) of age on action selection NRT; $a b$, indirect mediation effect (dashed arrows); $a$, the effect of age on prefronto-striatal connectivity; $b$, the effect of prefronto-striatal connectivity on action selection NRT while controlling for age; $c^{\prime}$, direct effect (dotted arrow) of age on action selection NRT. The mean (SE) path coefficient is shown. Bold rectangles represent significant mediators. $* * * p<0.001 . * p<0.05$. 
only two extreme age groups were compared (Boisgontier et al., 2016). Additionally, a significant effect of task complexity on both NRT and NER was found, whereby the 2L-Diag and 3L stimulus clusters were the most difficult ones. This is consistent with our coupling-decoupling model (Boisgontier et al., 2014), which defines recruitment and selection principles as defining parameters for multilimb action selection. On the one hand, the effector recruitment principle indicates that task complexity increases when the number of effectors to be moved increases (e. g., the $3 \mathrm{~L}$ stimulus cluster is more complex than the $2 \mathrm{~L}$ and $1 \mathrm{~L}$ cluster) (Jensen, 2006; Boisgontier et al., 2014). On the other hand, the effector selection principle states that the cognitive burden of moving a particular combination of limbs is dependent on preexisting coordination preferences (e.g., 2L-Diag is more complex than 2L-Homo cluster). While SRT is mainly linked to the recruitment principle, CRT results from a combination of recruitment and selection principles and is a better indicator of information processing time for action selection. Accordingly, the increased NRT (CRT - SRT) observed in older adults reflects a significant change in available neural resources required for central processing, giving rise to deficits in action selection. Furthermore, this age-related performance deterioration was more pronounced in highly demanding tasks, placing a higher burden on central processing.

Regarding the error rates, no age-related differences were found. Moreover, NER was not associated with NRT and controlling for NER in the SC-NRT analysis yielded similar results (data not shown). Presumably, OAs compared with YAs were differently positioned on the speed-accuracy trade-off continuum by sacrificing reaction speed for higher accuracy (Starns and Ratcliff, 2010). In other words, older participants appeared to increase RT to preserve high accuracy levels.

\section{Effects of age on SC}

Both white and gray matter structure is altered with aging. Here we focused on white matter connectivity between prefrontal regions and basal ganglia nuclei. In older adults, we found a significant decline in prefronto-striatal white matter connectivity, particularly for connections with CA nucleus and NAC. Many studies have used dMRI to study age effects on white matter connections (O'Sullivan et al., 2001; Sullivan and Pfefferbaum, 2006; Bennett et al., 2010; Zahr et al., 2010; de Groot et al., 2016), including frontal or striatal connections (Bennett et al., 2010; Burzynska et al., 2010). More specifically, a combined measure of functional and structural prefronto-striatal connectivity was found to be a prominent predictor of chronological age (Bonifazi et al., 2018).

With respect to gray matter, volume and shape analysis of subcortical structures have revealed significant atrophy and deformations in subcortical areas (including thalamus, putamen, $\mathrm{CA}$, and pallidum) with behavioral consequences in older adults. For example, subregional thalamic atrophy was associated with sensorimotor performance decline in older adults (Serbruyns et al., 2015), whereas subregional shape of right CA, putamen, NAC, and thalamus was found to be associated with motor learning capability (Chalavi et al., 2018). Furthermore, NAC atrophy in older adults was found to be associated with longer action selection times in the ML-RT (Boisgontier et al., 2016). Together, these studies indicate that age-related differences in subcortical gray matter are associated with motor deficits.

White matter studies have primarily used diffusion tensor imaging measures, such as fractional anisotropy and mean diffusivity. Here, we made use of 3-tissue constrained spherical deconvolution, which provides a better estimation of fiber orientations and tissue composition in white matter voxels (Jeurissen et al., 2014; Dhollander et al., 2017). Hence, it overcomes the inadequacy of diffusion tensor imaging in up to $\sim 90 \%$ of white matter voxels containing crossing fibers (Jeurissen et al., 2013). Moreover, we used anatomically constrained tractography (Smith et al., 2012) to constrain streamlines to connect gray matter regions and spherical-deconvolution informed filtering of tractograms (SIFT) (Smith et al., 2015a,b) to fit connection weights to the dMRI data. The total weight for connections obtained by this method has been proposed as a marker of white matter total cross-sectional volume (Smith et al., 2015b). The age-related decline in connection weight that we observed might be because of a reduction of axonal diameter or axon deletion, as suggested by postmortem histologic studies (Meier-Ruge et al., 1992; Tang et al., 1997; Marner et al., 2003; Peters and Rosene, 2003). However, it is currently unknown how well the assumptions of the SIFT method hold in the presence of the large proportion of false positive streamlines common for whole-brain probabilistic tractography (Maier-Hein et al., 2017): the SIFT method performs a global optimization of all connection weights, where false positives impact on genuine connection weights. SIFT results should be interpreted with caution (Zalesky et al., 2020).

\section{Effects of age on brain-behavior associations}

Our results revealed negative associations between left NAC-left rostral medial frontal gyrus, left NAC-left FP, right NAC-right rostral medial frontal gyrus, and right NAC-right FP connectivity and RT (NRT) in easy conditions as well as between right CA-right FP and left CA-right FP connectivity and increased NRT in difficult conditions. Interestingly, SC of left NACleft rostral medial frontal gyrus and left CA-right FP was found to significantly mediate the relationship between age and NRT under easy and difficult task conditions, respectively. Together, these findings suggest that reduced prefronto-striatal connectivity might compromise information transfer, resulting in slower action selection times in older adults.

NAC plays a prominent role in our task. Although it is a central node in the reward circuit (Purves et al., 2014), its role in action selection has also been documented. Not surprisingly, administration of dopamine to NAC has been found to benefit action selection performance (Nicola, 2007). Similarly, a significant negative association was found between SC of the CA with prefrontal regions and NRT in the difficult ML-RT condition in the present study. Previous work has already shown negative associations between gray matter atrophy of the CA and NRT in the more complex ML-RT subtasks (Boisgontier et al., 2016).

With respect to the prefrontal regions, connectivity of the RMFG and FP with striatum was predictive of NRT. Because an optimal balance between excitatory and inhibitory mechanisms is required for effector selection in the ML-RT task, it is not surprising that the prefrontal areas are figuring prominently because of their involvement in cognitive control functions. The FP is more activated in older adults in an anti-saccade RT task that requires suppression of preferred response tendencies (Fernandez-Ruiz et al., 2018). Moreover, the FP is also endowed with cognitive branching and monitoring of action outcomes (Koechlin, 2011) which are important ML-RT task functions.

Finally, SC of striatum with rostral medial frontal gyrus (DLPFC) showed a significant association with ML-RT 
performance. DLPFC is involved in various executive/cognitive control processes, including working memory (Kass and Mizrahi, 2010), action inhibition (Coxon et al., 2016), attention (Sylvester et al., 2003), task switching (Coxon et al., 2010, 2016), and planning. In complex interlimb coordination tasks, compensatory DLPFC recruitment has frequently been observed in OAs versus YAs (Heuninckx et al., 2005, 2008). These DLPFC functions are also relevant for the ML-RT task, particularly in relation to preexisting intereffector interactions that either support or compromise effector selection and integration. For instance, strong coupling exists between the homologous effectors, and this promotes fast movements of both hands or both feet together (Boisgontier et al., 2014). Conversely, in the most difficult 2L Diag and 3L subtasks, participants need to inhibit one or both contralateral homologous limbs. Similarly, DLPFC is also active during conflict monitoring and/or inhibitory control, as evaluated by Go/NoGo and Flanker tasks (Casey et al., 2000; Watanabe et al., 2002). Additionally, in a stop signal RT task, we observed overactivation of DLPFC during performance of the "Go trials" in older adults (Coxon et al., 2016). Together, these findings suggest a prominent role of DLPFC in processes of complex action selection. The reduced connectivity between DLPFC and striatum in older adults may trigger DLPFC overactivation in older adults (Coxon et al., 2010), possibly as compensation for prefronto-striatal pathway deficits. This hypothesis requires further investigation.

\section{Limitations}

Action selection errors in ML-RT can be categorized into two types: commission errors (i.e., lifting a limb that should stay down) and omission errors (i.e., not lifting a limb that should be lifted). Our task setup did not detect omission error trials. However, since such trials would normally have unusually high RT values, our outlier detection method most likely removed such error trials from the RT analysis.

In conclusion, aging is characterized by a significant decline in action selection performance that is associated with reduced prefronto-striatal SC. Preserving this connectivity may be an important target for intervention to promote healthy aging and independent living. More broadly, changes occurring in the aging brain are complex and multifactorial referring to alterations in brain function, structure, connectivity, and neurochemicals. A multimodal approach appears most promising to unveil such complex brain-behavioral interactions.

\section{References}

Alexander GE, DeLong MR, Strick PL (1986) Parallel organization of functionally segregated circuits linking basal ganglia and cortex. Annu Rev Neurosci 9:357-381.

Andersson JL, Sotiropoulos SN (2016) An integrated approach to correction for off-resonance effects and subject movement in diffusion MR imaging. Neuroimage 125:1063-1078.

Andersson JL, Skare S, Ashburner J (2003) How to correct susceptibility distortions in spin-echo echo-planar images: application to diffusion tensor imaging. Neuroimage 20:870-888.

Andersson JL, Graham MS, Zsoldos E, Sotiropoulos SN (2016) Incorporating outlier detection and replacement into a non-parametric framework for movement and distortion correction of diffusion MR images. Neuroimage 141:556-572.

Baron RM, Kenny DA (1986) The moderator-mediator variable distinction in social psychological research: conceptual, strategic, and statistical considerations. J Pers Soc Psychol 51:1173-1182.

Bartlett MS (1947) The use of transformations. Biometrics 3:39-52.
Benjamini Y, Hochberg Y (1995) Controlling the false discovery rate: a practical and powerful approach to multiple testing. J R Stat Soc B 57:289300 .

Bennett IJ, Madden DJ, Vaidya CJ, Howard DV, Howard JJ (2010) Agerelated differences in multiple measures of white matter integrity: a diffusion tensor imaging study of healthy aging. Hum Brain Mapp 31:378390.

Boisgontier MP, Wittenberg GF, Fujiyama H, Levin O, Swinnen SP (2014) Complexity of central processing in simple and choice multilimb reaction-time tasks. PLoS One 9:e90457.

Boisgontier MP, van Ruitenbeek P, Leunissen I, Chalavi S, Sunaert S, Levin O, Swinnen SP (2016) Nucleus accumbens and caudate atrophy predicts longer action selection times in young and old adults. Hum Brain Mapp 37:4629-4639.

Bonifazi P, Erramuzpe A, Diez I, Gabilondo I, Boisgontier MP, Pauwels L, Stramaglia S, Swinnen SP, Cortes JM (2018) Structure-function multiscale connectomics reveals a major role of the fronto-striato-thalamic circuit in brain aging. Hum Brain Mapp 39:4663-4677.

Burzynska AZ, Preuschhof C, Bäckman L, Nyberg L, Li SC, Lindenberger U, Heekeren HR (2010) Age-related differences in white matter microstructure: region-specific patterns of diffusivity. Neuroimage 49:2104-2112.

Cacciola A, Calamuneri A, Milardi D, Mormina E, Chillemi G, Marino S, Naro A, Rizzo G, Anastasi G, Quartarone A (2017) A connectomic analysis of the human basal ganglia network. Front Neuroanat 11:85.

Casey B, Thomas KM, Welsh TF, Badgaiyan RD, Eccard CH, Jennings JR, Crone EA (2000) Dissociation of response conflict, attentional selection, and expectancy with functional magnetic resonance imaging. Proc Natl Acad Sci USA 97:8728-8733.

Chalavi S, Adab HZ, Pauwels L, Beets IA, van Ruitenbeek P, Boisgontier MP, Monteiro TS, Maes C, Sunaert S, Swinnen SP (2018) Anatomy of subcortical structures predicts age-related differences in skill acquisition. Cereb Cortex 28:459-473.

Cisek P (2007) Cortical mechanisms of action selection: the affordance competition hypothesis. Philos Trans R Soc Lond B Biol Sci 362:1585-1599.

Colder B (2015) The basal ganglia select the expected sensory input used for predictive coding. Front Comput Neurosci 9:119.

Coxon JP, Goble DJ, Van Impe A, De Vos J, Wenderoth N, Swinnen SP (2010) Reduced basal ganglia function when elderly switch between coordinated movement patterns. Cereb Cortex 20:2368-2379.

Coxon JP, Goble DJ, Leunissen I, Van Impe A, Wenderoth N, Swinnen SP (2016) Functional brain activation associated with inhibitory control deficits in older adults. Cereb Cortex 26:12-22.

de Groot M, Cremers LG, Ikram MA, Hofman A, Krestin GP, van der Lugt A, Niessen WJ, Vernooij MW (2016) White matter degeneration with aging: longitudinal diffusion MR imaging analysis. Radiology 279:532541.

Desikan RS, Ségonne F, Fischl B, Quinn BT, Dickerson BC, Blacker D, Buckner RL, Dale AM, Maguire RP, Hyman BT, Albert MS, Killiany RJ (2006) An automated labeling system for subdividing the human cerebral cortex on MRI scans into gyral based regions of interest. Neuroimage 31:968-980.

Dhollander T, Raffelt D, Connelly A (2016) Unsupervised 3-tissue response function estimation from single-shell or multi-shell diffusion MR data without a co-registered T1 image. In: ISMRM Workshop on Breaking the Barriers of Diffusion MRI, p 5, Lisbon, Portugal.

Dhollander T, Raffelt D, Connelly A (2017) Towards interpretation of 3-tissue constrained spherical deconvolution results in pathology. Proc Intl Soc Mag Reson Med, p 1815.

Donders FC (1969) On the speed of mental processes. Acta Psychol (Amst) 30:412-431.

Fernandez-Ruiz J, Peltsch A, Alahyane N, Brien DC, Coe BC, Garcia A, Munoz DP (2018) Age related prefrontal compensatory mechanisms for inhibitory control in the antisaccade task. Neuroimage 165:92-101.

Fischl B (2012) FreeSurfer. Neuroimage 62:774-781.

Greenhouse I, Sias A, Labruna L, Ivry RB (2015) Nonspecific inhibition of the motor system during response preparation. J Neurosci 35:1067510684 .

Gurney KN, Prescott TJ, Redgrave P (1998) The basal ganglia viewed as an action selection device. In: International Conference on Artificial Neural Networks, p 1033-1038. 
Heuninckx S, Wenderoth N, Swinnen SP (2008) Systems neuroplasticity in the aging brain: recruiting additional neural resources for successful motor performance in elderly persons. J Neurosci 28:91-99.

Heuninckx S, Wenderoth N, Debaere F, Peeters R, Swinnen SP (2005) Neural basis of aging: the penetration of cognition into action control. J Neurosci 25:6787-6796.

Humphries MD (2014) Basal ganglia: mechanisms for action selection. In: Encyclopedia of Computational Neuroscience. New York, NY: Springer.

Jenkinson M, Beckmann CF, Behrens TE, Woolrich MW, Smith SM (2012) Fsl. Neuroimage 62:782-790.

Jensen AR (2006) Clocking the mind: mental chronometry and individual differences. Amsterdam: Elsevier.

Jeurissen B, Leemans A, Tournier JD, Jones DK, Sijbers J (2013) Investigating the prevalence of complex fiber configurations in white matter tissue with diffusion magnetic resonance imaging. Hum Brain Mapp 34:2747-2766.

Jeurissen B, Tournier JD, Dhollander T, Connelly A, Sijbers J (2014) Multitissue constrained spherical deconvolution for improved analysis of multi-shell diffusion MRI data. Neuroimage 103:411-426.

Kass JS, Mizrahi EM (2010) Neurology secrets e-book. Amsterdam: Elsevier.

Koechlin E (2011) Frontal pole function: what is specifically human? Trends Cogn Sci 15:241.

Labruna L, Lebon F, Duque J, Klein PA, Cazares C, Ivry RB (2014) Generic inhibition of the selected movement and constrained inhibition of nonselected movements during response preparation. J Cogn Neurosci 26:269-278.

Leys C, Ley C, Klein O, Bernard P, Licata L (2013) Detecting outliers: do not use standard deviation around the mean, use absolute deviation around the median. J Exp Soc Psychol 49:764-766.

MacKinnon DP, Fairchild AJ, Fritz MS (2007) Mediation analysis. Annu Rev Psychol 58:593-614.

Maier-Hein KH, Neher PF, Houde JC, Côté MA, Garyfallidis E, Zhong J, Chamberland M, Yeh FC, Lin YC, Ji Q, Reddick WE, Glass JO, Chen DQ, Feng Y, Gao C, Wu Y, Ma J, He R, Li Q, Westin CF, et al. (2017) The challenge of mapping the human connectome based on diffusion tractography. Nat Commun 8:13.

Marner L, Nyengaard JR, Tang Y, Pakkenberg B (2003) Marked loss of myelinated nerve fibers in the human brain with age. J Comp Neurol 462:144-152.

Meier-Ruge W, Ulrich J, Brühlmann M, Meier E (1992) Age-related white matter atrophy in the human brain. Ann NY Acad Sci 673:260-269.

Milardi D, Quartarone A, Bramanti A, Anastasi G, Bertino S, Basile GA, Buonasera P, Pilone G, Celeste G, Rizzo G, Bruschetta D, Cacciola A (2019) The cortico-basal ganglia-cerebellar network: past, present and future perspectives. Front Syst Neurosci 13:61.

Miller EK (2000) The prefontral cortex and cognitive control. Nat Rev Neurosci 1:59-65.

Mink JW (1996) The basal ganglia: focused selection and inhibition of competing motor programs. Prog Neurobiol 50:381-425.

Nasreddine ZS, Phillips NA, Bédirian V, Charbonneau S, Whitehead V, Collin I, Cummings JL, Chertkow H (2005) The Montreal Cognitive Assessment, MoCA: a brief screening tool for mild cognitive impairment. J Am Geriatr Soc 53:695-699.

Nicola SM (2007) The nucleus accumbens as part of a basal ganglia action selection circuit. Psychopharmacology (Berl) 191:521-550.

O'Sullivan M, Jones DK, Summers PE, Morris RG, Williams SC, Markus HS (2001) Evidence for cortical "disconnection" as a mechanism of agerelated cognitive decline. Neurology 57:632-638.

Patenaude B, Smith SM, Kennedy DN, Jenkinson M (2011) A Bayesian model of shape and appearance for subcortical brain segmentation. Neuroimage 56:907-922.

Peters A, Rosene DL (2003) In aging, is it gray or white? J Comp Neurol 462:139-143.

Purves D, Augustine GJ, Fitzpatrick D, Hall W, LaMantia A, McNamara J, White L (2014) Neuroscience, pp 15-16. Sunderland, MA: Sinauer.

Redgrave P, Prescott TJ, Gurney K (1999) The basal ganglia: a vertebrate solution to the selection problem? Neuroscience 89:1009-1023.

Rubinov M, Sporns O (2010) Complex network measures of brain connectivity: uses and interpretations. Neuroimage 52:1059-1069.

Schultz W (2004) Neural coding of basic reward terms of animal learning theory, game theory, microeconomics and behavioural ecology. Curr Opin Neurobiol 14:139-147.
Schultz W, Tremblay L, Hollerman JR (2000) Reward processing in primate orbitofrontal cortex and basal ganglia. Cereb Cortex 10:272-283.

Serbruyns L, Leunissen I, Huysmans T, Cuypers K, Meesen RL, Van Ruitenbeek P, Sijbers J, Swinnen SP (2015) Subcortical volumetric changes across the adult lifespan: subregional thalamic atrophy accounts for age-related sensorimotor performance declines. Cortex 65:128-138.

Sink CA, Stroh HR (2006) Practical significance: the use of effect sizes in school counseling research. Prof School Counsel 9:401-411.

Smith RE, Tournier JD, Calamante F, Connelly A (2012) Anatomically-constrained tractography: improved diffusion MRI streamlines tractography through effective use of anatomical information. Neuroimage 62:19241938.

Smith RE, Tournier JD, Calamante F, Connelly A (2015a) The effects of SIFT on the reproducibility and biological accuracy of the structural connectome. Neuroimage 104:253-265.

Smith RE, Tournier JD, Calamante F, Connelly A (2015b) SIFT2: enabling dense quantitative assessment of brain white matter connectivity using streamlines tractography. Neuroimage 119:338-351.

Starns JJ, Ratcliff R (2010) The effects of aging on the speed-accuracy compromise: boundary optimality in the diffusion model. Psychol Aging 25:377-390.

Stoloff RH, Taylor JA, Xu J, Ridderikhoff A, Ivry RB (2011) Effect of reinforcement history on hand choice in an unconstrained reaching task. Front Neurosci 5:41.

Sullivan EV, Pfefferbaum A (2006) Diffusion tensor imaging and aging. Neurosci Biobehav Rev 30:749-761.

Swinnen SP (2002) Intermanual coordination: from behavioural principles to neural-network interactions. Nat Rev Neurosci 3:348-359.

Sylvester CY, Wager TD, Lacey SC, Hernandez L, Nichols TE, Smith EE, Jonides J (2003) Switching attention and resolving interference: fMRI measures of executive functions. Neuropsychologia 41:357-370.

Tang Y, Nyengaard J, Pakkenberg B, Gundersen H (1997) Age-induced white matter changes in the human brain: a stereological investigation. Neurobiol Aging 18:609-615.

Tanji J, Hoshi E (2001) Behavioral planning in the prefrontal cortex. Curr Opin Neurobiol 11:164-170.

Tournier JD, Smith R, Raffelt D, Tabbara R, Dhollander T, Pietsch M, Christiaens D, Jeurissen B, Yeh CH, Connelly A (2019) MRtrix3: a fast, flexible and open software framework for medical image processing and visualisation. Neuroimage 202:116137.

Tournier JD, Calamante F, Connelly A (2010) Improved probabilistic streamlines tractography by 2 nd order integration over fibre orientation distributions. In: Proceedings of the International Society for Magnetic Resonance in Medicine.

Veraart J, Novikov DS, Christiaens D, Ades-Aron B, Sijbers J, Fieremans E (2016) Denoising of diffusion MRI using random matrix theory. Neuroimage 142:394-406.

Wager TD, Davidson ML, Hughes BL, Lindquist MA, Ochsner KN (2008) Prefrontal-subcortical pathways mediating successful emotion regulation. Neuron 59:1037-1050.

Wager TD, Waugh CE, Lindquist M, Noll DC, Fredrickson BL, Taylor SF (2009) Brain mediators of cardiovascular responses to social threat: I. Reciprocal dorsal and ventral sub-regions of the medial prefrontal cortex and heart-rate reactivity. Neuroimage 47:821-835.

Wallis JD (2007) Orbitofrontal cortex and its contribution to decision-making. Annu Rev Neurosci 30:31-56.

Watanabe J, Sugiura M, Sato K, Sato Y, Maeda Y, Matsue Y, Fukuda H, Kawashima R (2002) The human prefrontal and parietal association cortices are involved in NO-GO performances: an event-related fMRI study. Neuroimage 17:1207-1216.

Welford A (1980) Choice reaction time: basic concepts. Reaction Times 73-128.

Wong AL, Haith AM, Krakauer JW (2015) Motor planning. Neuroscientist 21:385-398.

Zahr NM, Pfefferbaum A, Sullivan EV (2010) Changes in the macrostructure and microstructure of the ageing brain. In: Principles and practice of geriatric psychiatry, pp 15-27. London: Wiley-Blackwell.

Zalesky A, Sarwar T, Ramamohanarao K (2020) A cautionary note on the use of SIFT in pathological connectomes. Magn Reson Med 83:791-794. 